\title{
Sizing Up Outer Billiard Tables
}

\author{
Filiz Doğru \\ Department of Mathematics \\ Grand Valley State University \\ 1 Campus Drive \\ Allendale, Michigan 49401 USA \\ Samuel Otten \\ Division of Science \& Mathematics Education \\ Michigan State University \\ 116 North Kedzie Hall \\ East Lansing, Michigan 48824 USA
}

Received: September 15, 2009

Accepted: March 16, 2010

\section{ABSTRACT}

The outer billiard dynamical system models the motion of a particle around a compact domain, such as a planet orbiting a star. When considering outer billiards in hyperbolic space, an interesting problem is to determine precisely the conditions in which an orbiting particle breaks orbit and escapes to infinity. Past work has classified triangular and Penrose kite billiard tables according to whether or not their orbiting particles escape. This article presents a classification of regular polygonal tables.

\section{INTRODUCTION}

If you mention billiards in casual conversation, you will likely elicit thoughts of a cue game that involves caroming balls on a cloth-covered table. If you happen to be conversing with a mathematician, however, the term may instead bring to mind the wellknown dynamical system involving the motion of a particle within a closed domain. The billiard dynamical system has a great deal of intrinsic beauty (e.g., see Figure 1), is a source of mathematical connections (e.g., to number theory [1] and Cantor sets [6]), and has applications to phenomena like the classical motion of gas particles in a closed container [12], optics, and quantum chaos [5]. Even non-mathematicians may be interested in the mathematical version of billiards when they learn that Lewis Carroll investigated billiard trajectories within certain polyhedra [14].

What if we move the game of billiards outside the table? This is, in fact, not a new idea but was first put forth fifty years ago by Bernhard Neumann [9].
Whereas inner billiards can be thought of as a crude model for the motion of gas particles within a closed region, outer billiards was proposed as a crude model for the motion of celestial objects within a gravitational field. Jürgen Moser popularized this idea when he related outer billiards to the stability problem [8] — will the planets of our solar system ever escape the gravitational pull of the sun? Correspondingly, are there any outer billiard systems from which things escape to infinity? This is known as the MoserNeumann problem.

The current paper, after a formal definition of the outer billiard transformation and a summary of a few known results, will continue the work on the Moser-Neumann problem by classifying regular polygonal tables in the hyperbolic plane.

\section{THE OUTER BILLIARD TRANSFORMATION}

Let $P$ be a convex billiard table, that is, a compact domain with piecewise smooth boundary. For inner billiards, one considers 


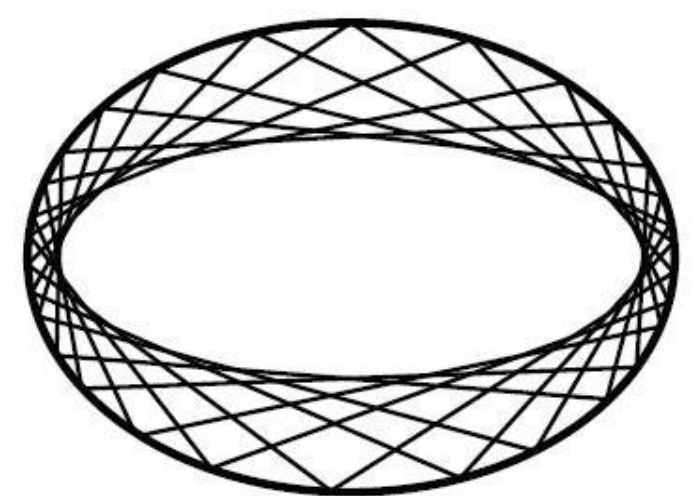

Figure 1. An elliptic billiard table with an elliptic envelope.

a ball in the interior of $P$ that reflects off the boundary curve. For outer billiards, we consider a ball in the exterior of $P$ that reflects through points on the boundary curve. (Outer billiards is referred to by some as dual billiards.) To be precise, given a table $P$ and a ball $x$ in the exterior, there are two points on $P$ that form support lines through $x$. The outer billiard transformation, denoted $F$, has either a clockwise or counterclockwise orientation and is defined two-dimensionally as the map that sends $x$ to its reflection through the support point in the given direction (see Figure 2).

This definition holds whenever the support point in the given direction is unique; $F(x)$ is not defined when $x$ lies on what is referred to as a discontinuity line (which is similar to the undefined nature of the inner billiard transformation when the ball hits a corner of the table). The map $F$ can be iterated and the set of all iterations of a particular point is called an orbit. If a point can be iterated so that it arrives back upon itself we say that the point and its orbit are periodic; that is, if $F^{k}(x)=x$ for some natural number $k$, and $k$ is the smallest such number, then $x$ and its orbit are $k$-periodic.

With regard to the Moser-Neumann problem, early progress was made by Moser himself who found that all orbits were bounded for sufficiently smooth tables [7]. Later, it was determined that quasirational polygonal tables also had the property that all orbits were bounded [3], [13]. A significant breakthrough was made by Schwartz who found that Penrose kite polygonal tables had unbounded orbits [11].

As in [2], we will be working specifically with polygonal tables in the hyperbolic plane where the outer billiard transformation can be defined by simply taking all geometric notions to be in their hyperbolic sense (e.g., reflecting points using hyperbolic distances). We employ the Beltrami-Klein disk model of the hyperbolic plane because of its intuitive representation of collinear points, though it should be noted that angles are non-conformal in this model. Within the Beltrami-Klein disk $F$ can be extended so as to be defined on the boundary of the disk. The restriction of $F$ to the boundary will be denoted $f$ and will play a large role in the work to follow. (Properties of the derivative of $f$ were previously explored in this journal [10].)

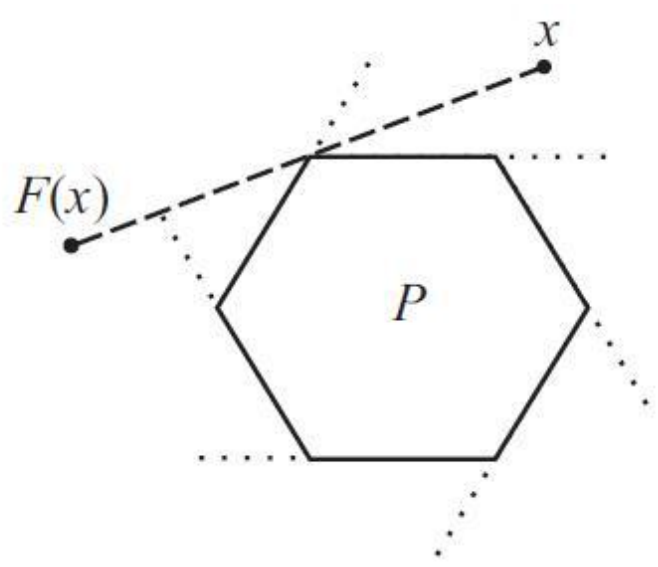

Figure 2. Outer billiard mappings with counterclockwise orientation. 


\section{TABLE SIZES}

The outer billiard map $F$ is an areaand orientation-preserving isometry (and in the case of polygonal tables, a piecewise isometry). Furthermore, since we are working in the hyperbolic plane, $F$ can be classified as an elliptic, parabolic, or hyperbolic isometry depending on the number and location of any periodic points. An elliptic isometry has one periodic point in the hyperbolic plane. A parabolic isometry has one periodic point at infinity, and a hyperbolic isometry has two periodic points at infinity.

The extended outer billiard map $f$ is a circle map and constitutes a rotation of the circle at infinity; more specifically, $f$ is an orientation-preserving circle homeomorphism [2]. Therefore, by a well-known result in dynamical systems, if a periodic orbit exists on the circle then all other periodic orbits of $f$ necessarily have the same period [4]. It is also the case that the existence of a single orbit on the circle (a parabolic isometry) implies that the points within this orbit are attractive on one side and repulsive on the other side. If two periodic orbits exist on the circle (a hyperbolic isometry), then the points from one orbit attract while the points from the other orbit repel. These descriptions, along with the elliptic case mentioned above, exhaust all possibilities for $f$ because it can have at most two periodic orbits on the circle [4]. (This limit to the number of periodic orbits at infinity is especially important in the next section.)

When dealing with a rotation on the circle such as $f$, it is common to consider the rotation number of the function, denoted $\rho(f)$. The rotation number can be thought of as the average portion of the circle that is traversed by one iteration of the transformation. Within outer billiards we are often concerned with the rotation number of a particular orbit, rather than of the transformation itself. This moves us to utilize the following notion of the geometric rotation number for a $\boldsymbol{q}$-periodic orbit $\boldsymbol{O}$, which will be denoted $\rho(\boldsymbol{O})$ and will refer to quotient of the winding number $\omega$ of the (possibly selfintersecting) polygon formed by $\boldsymbol{O}$ and the period of $\boldsymbol{O}$; that is, $\rho(\boldsymbol{O})=\omega / q$ (see Figure $3)$. This holds both for orbits of $F$ in the hyperbolic plane and orbits of $f$ at infinity.

What does all this have to do with the Moser-Neumann problem? The answer is that by using the mathematical framework above we can classify polygonal tables in the hyperbolic plane, and a table's classification will tell us whether or not its orbits escape to infinity. The classes-small, neutral, and large-are defined using geometric rotation numbers and the derivatives of periodic points at infinity. (The derivative of the outer billiard map in the hyperbolic plane is not central to the purposes of this article and so will not be developed here. See [2] and [10] for more.) A small table corresponds with an elliptic isometry and so has periodic points in the hyperbolic plane. A neutral table corresponds with a parabolic isometry and so has a single periodic orbit on the circle (which can be called a neutral orbit). A large table corresponds with a hyperbolic isometry and so has two periodic orbits on the circle. Since one of these orbits on the circle is necessarily attractive, all orbits around a
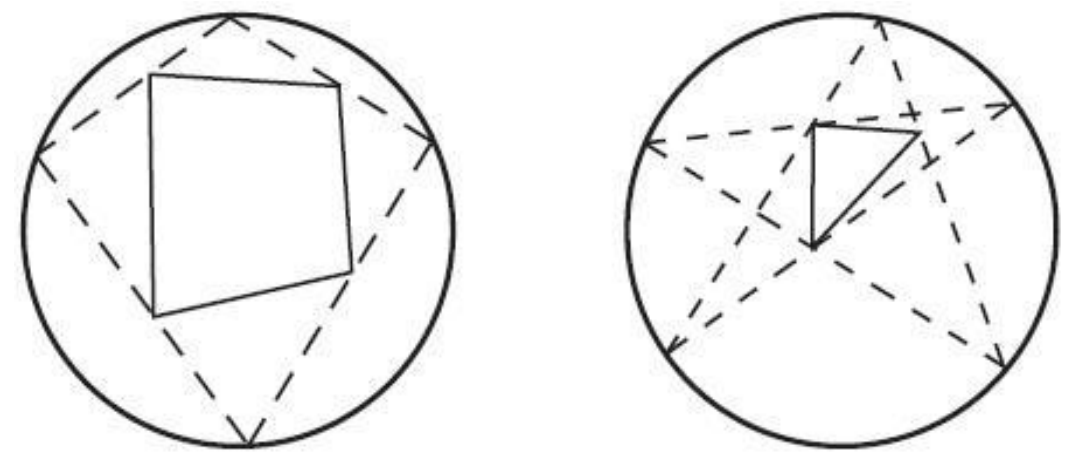

Figure 3. Orbits with rotation numbers $1 / 4$ and $2 / 5$, respectively. 
large table escape to infinity. This final fact is, of course, the motivation behind the classification.

The class of an $n$-gon table can be determined by locating a particular $n$ periodic orbit, namely, one with rotation number $1 / n$. We will use $\boldsymbol{N}$ to refer to this particular type of orbit. If $\boldsymbol{N}$ is in the interior of the hyperbolic plane, then the table is small. If $\boldsymbol{N}$ lies on the boundary circle, then the table is neutral. And if two such orbits lie on the boundary circle, then the table is necessarily large. Ideally, we would like to be able to classify a polygonal table simply using characteristics of the table itself, without having to search for and analyze any $n$-periodic orbits. Toward this end, we move on to the next section where we will briefly review the existing classification method for triangular tables and then introduce a new classification method for regular polygonal tables.

\section{CLASSIFYING REGULAR POLYGONAL TABLES}

Using area properties of triangles in the hyperbolic plane, a geometric description of large triangles was developed in terms of its sides, $a_{1}, a_{2}, a_{3}$, or its interior angles, $\alpha_{1}, \alpha_{2}, \alpha_{3}$ [2]. For example, a triangle is large if and only if

$$
\cos ^{2} \alpha_{1}+\cos ^{2} \alpha_{2}+\cos ^{2} \alpha_{3}+2 \cos \alpha_{1} \cos \alpha_{2} \cos \alpha_{3}>1+\sin \alpha_{1} \sin \alpha_{2} \sin \alpha_{3}
$$

As was mentioned in the previous section, the location of a particular type of orbit, denoted $\boldsymbol{N}$, is indicative of the classification of an $n$-gon table. It will be helpful to know precisely where to find such an orbit under our condition of interest-regular polygonal tables. The following lemma provides the specific location of such an orbit, if it exists, relative to the table.

Lemma. Let $F$ be the outer billiard map defined for a non-large, regular n-gon table $P$ with interior angle $\tau$. Let $s_{1}, s_{2}, \ldots, s_{n}$ be the vertices of $P$ labeled in order according to the orientation of $F$. If $x$ is the point from the exterior side of $s_{n} s_{1}$ that

(i) lies on the perpendicular bisector of $s_{n} s_{1}$, and

(ii) forms $\angle x s_{1} s_{n}=(\pi-\tau) / 2$,

then $x$ is an element of the unique $n$-periodic orbit $N$ with rotation number $1 / n$. Furthermore, $\mathbf{N}$ forms a regular $n$-gon $\mathbf{N}$ with interior angle $\phi<\tau$.

By the definition of the outer billiard map, $\left|x s_{1}\right|=\left|F(x) s_{1}\right|$. Since $P$ is a regular polygon, $\left|s_{n} s_{1}\right|=\left|\begin{array}{ll}s_{2} & s_{1}\end{array}\right|$, and by our assumption, $\angle x s_{1} s_{n}=(\pi-\tau) / 2$. Let us consider $\angle F(x) s_{1} s_{2}$. Since we have three supplementary angles,

$$
\angle F(x) s_{1} s_{2}=\pi-\tau-\frac{\pi-\tau}{2}=\frac{\pi-\tau}{2},
$$

and thus $\angle x s_{1} s_{n}=\angle F(x) s_{1} s_{2}$ (see Figure 4 , for example). By side-angle-side we have congruent triangles, $x s_{1} s_{n} \cong F(x) s_{1} s_{2}$. (Note that SAS is a valid congruence condition in hyperbolic geometry.)

Since $x$ lies on the perpendicular bisector of $s_{n} s_{1}$, it must be the case that $\left|x s_{1}\right|=\left|x s_{n}\right|$ which implies via congruence that $\left|F(x) s_{1}\right|=\left|F(x) s_{2}\right|$. By the isosceles triangle theorem, we have $\angle F(x) s_{2} s_{1}=(\pi-\tau) / 2$. We can now mimic the argument for congruent triangles with $F(x)$ and $F^{2}(x)$ in place of $x$ and $F(x)$, respectively. Continuing around the table, we find triangles $x s_{1} s_{n} \cong F^{i}(x) s_{i} s_{i+1}$ for $1 \leq$ $i<n$. This means that the points $F^{n-1}(x)$ and $x$ will be equidistant from $s_{n}$. Furthermore, since

$$
\angle F^{n-1}(x) s_{n} s_{n-1}+\tau+\angle x s_{n} s_{1}=\frac{\pi-\tau}{2}+\tau+\frac{\pi-\tau}{2}=\pi,
$$




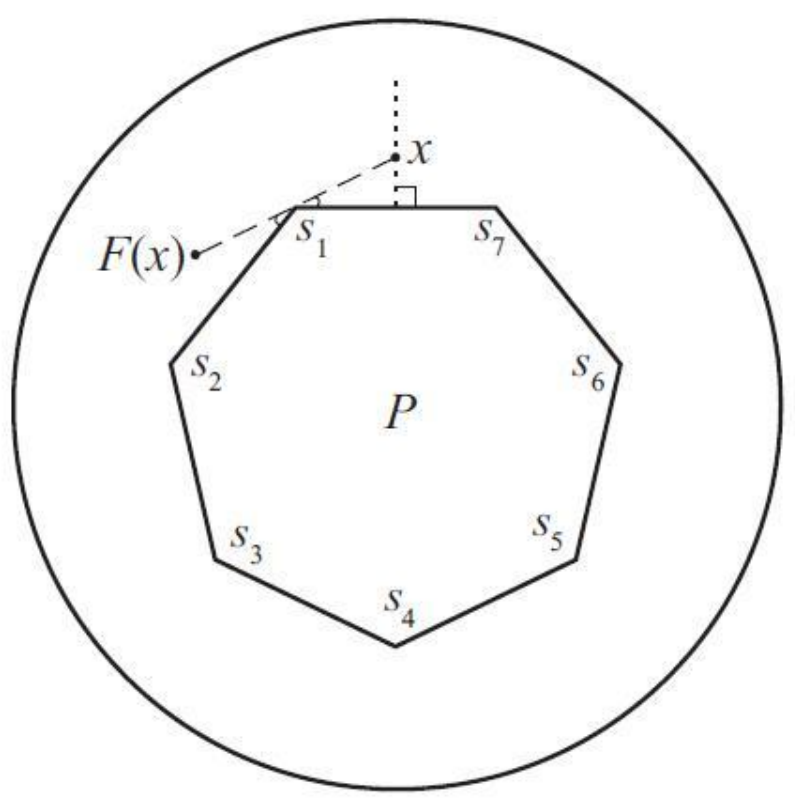

Figure 4. A regular 7-gon table and the point $x$.

we know that $F^{n-1}(x), s_{n}$, and $x$ are collinear. Therefore, $F^{n}(x)=x$ and the orbit $\boldsymbol{N}$ is $n$ periodic with $\rho(\boldsymbol{N})=1 / n$. Since $P$ is a nonlarge table, $F$ must be an elliptic or parabolic isometry, either of which guarantees that $\boldsymbol{N}$ is unique. The rest of the lemma can be verified forthwith.

We have determined that the periodic orbit $\boldsymbol{N}$, which for regular polygonal tables is significantly described in the lemma, can serve as an indicator of the table's classification (see Figure 5). It should also be noted that the neutral case serves as the key to the general classification-a bifurcation point, in a sense-because small and large tables can be considered with regard to the neutral condition. In other words, the small and large cases can be revealed through knowledge of the neutral case. This observation will play a significant role in the argument below.

Now let us take an even closer look at the relationship between a regular $n$-gon table $P$ and its orbit $\boldsymbol{N}$. Viewing this orbit as the closed figure $N$, we know from the lemma that $N$ itself is also a regular $n$-gon.

Let $P$ be a small regular polygonal table. We can construct a triangle by joining the center of $P$ with two consecutive vertices of $P$. By the regularity of the table, this triangle will have two angles of $\tau / 2$ and one angle of $2 \pi / n$. (Note that in hyperbolic geometry $\tau<\pi(n-2) / n$.) Letting a be the length of a side of $P$, we have from the dual hyperbolic law of cosines

$$
\cosh a=\frac{\cos ^{2}(\tau / 2)+\cos (2 \pi / n)}{\sin ^{2}(\tau / 2)}
$$

This side of $P$ also defines a triangle inside $\boldsymbol{N}$ (which exists because $P$ is small). Let $\phi$ be the interior angle of $N$ (see Figure $6)$. By the lemma we have $(\pi-\tau) / 2$ as two angles of this triangle, with $\phi$ as the third. Thus, within $N$ we get

$$
\cosh a=\frac{\cos ^{2}((\pi-\tau) / 2)+\cos \phi}{\sin ^{2}((\pi-\tau) / 2)} .
$$

We can simplify the right-hand side by using the trigonometric rule of complements wherever we have a sine or cosine of $(\pi-\tau) / 2=\pi / 2-\tau / 2$, leaving

$$
\cosh a=\frac{\sin ^{2}(\tau / 2)+\cos \phi}{\cos ^{2}(\tau / 2)} .
$$



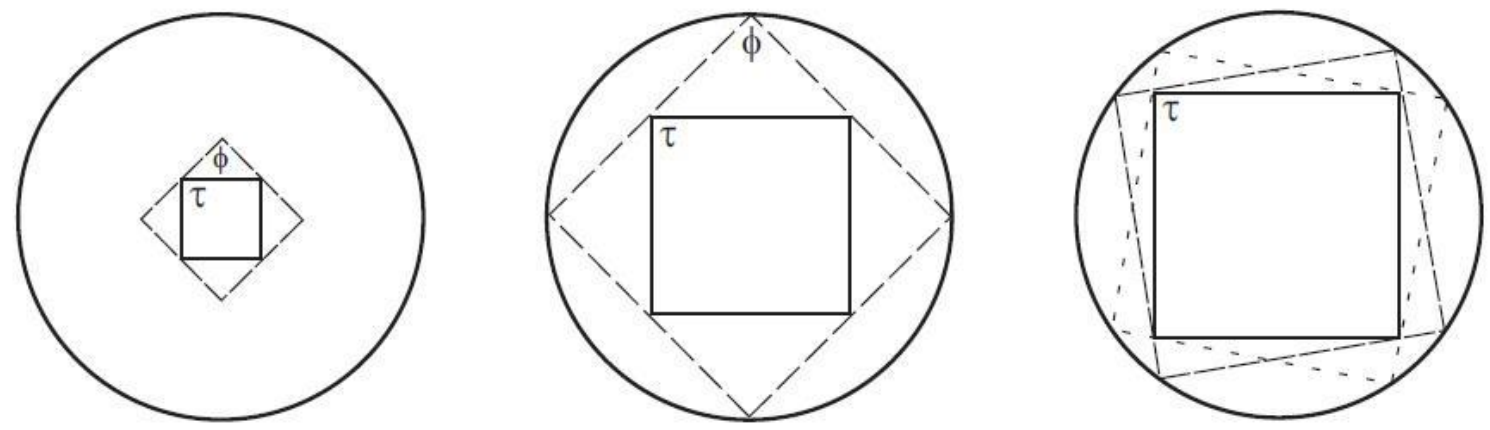

Figure 5. Small, neutral, and large regular 4-gon tables in the Klein-Beltrami model.

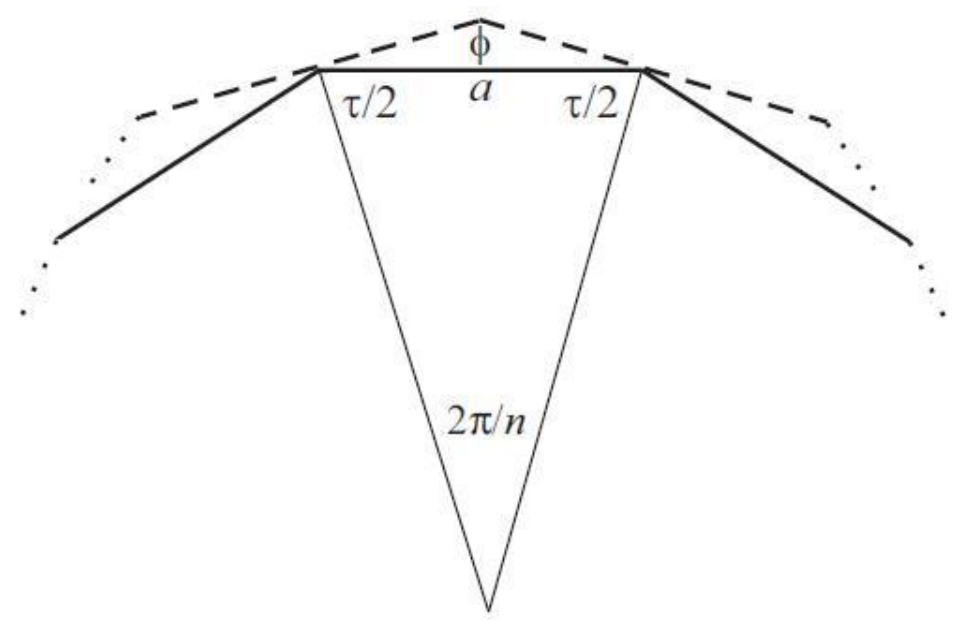

Figure 6. Two triangles sharing the side a.

We substitute this expression into Equation 1 and find that

$$
\frac{\sin ^{2}(\tau / 2)+\cos \phi}{\cos ^{2}(\tau / 2)}=\frac{\cos ^{2}(\tau / 2)+\cos (2 \pi / n)}{\sin ^{2}(\tau / 2)}=\frac{\cos ^{2}(\tau / 2)}{\sin ^{2}(\tau / 2)}+\frac{\cos (2 \pi / n)}{\sin ^{2}(\tau / 2)}
$$

Since $\phi$ is dependent upon $\tau$, it makes sense to simplify so that

$$
\begin{aligned}
\cos \phi & =\frac{\cos ^{4}(\tau / 2)}{\sin ^{2}(\tau / 2)}-\sin ^{2}(\tau / 2)+\frac{\cos ^{2}(\tau / 2) \cdot \cos (2 \pi / n)}{\sin ^{2}(\tau / 2)} \\
& =\frac{\cos ^{4}(\tau / 2)-\sin ^{4}(\tau / 2)}{\sin ^{2}(\tau / 2)}+\cot ^{2}(\tau / 2) \cdot \cos (2 \pi / n)
\end{aligned}
$$

We have the difference of perfect squares in the numerator, so 

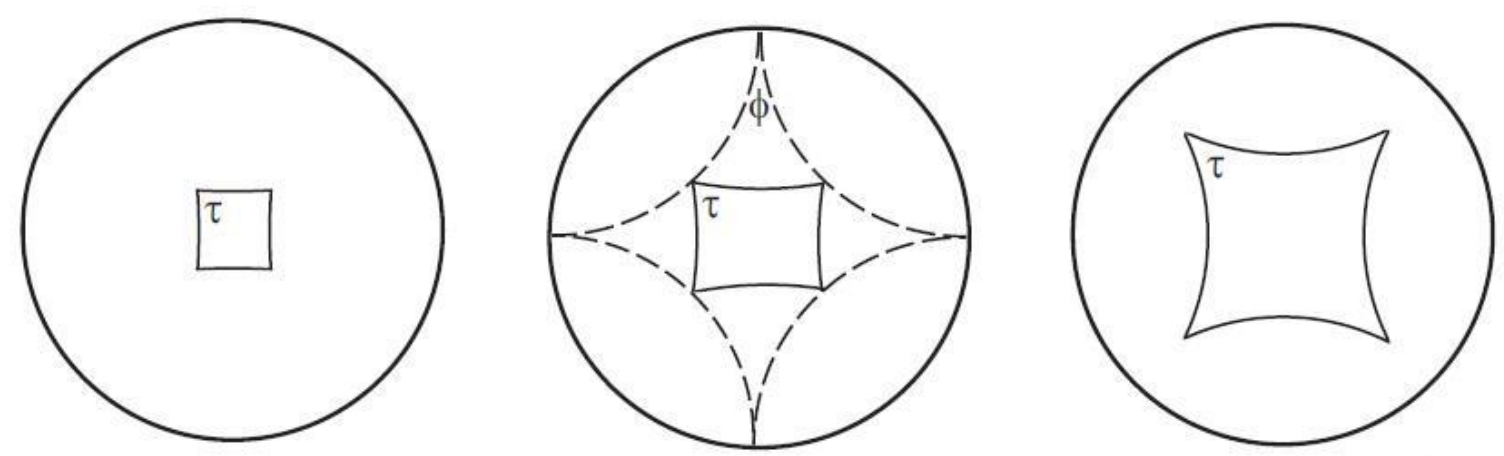

Figure 7. Small, neutral, and large regular 4-gon tables in the Poincaré model of the hyperbolic plane

$$
\begin{aligned}
\cos \phi & =\frac{\cos ^{2}(\tau / 2)-\sin ^{2}(\tau / 2)}{\sin ^{2}(\tau / 2)}+\cot ^{2}(\tau / 2) \cdot \cos (2 \pi / n) \\
& =\cot ^{2}(\tau / 2)-1+\cot ^{2}(\tau / 2) \cdot \cos (2 \pi / n) \\
& =\cot ^{2}(\tau / 2)(1+\cos (2 \pi / n))-1
\end{aligned}
$$

Now we let the size of $P$ vary until $\boldsymbol{N}$ is precisely on the boundary of the hyperbolic model. This would occur when the interior angle of $N$ reaches 0 . Substituting 0 in for $\phi$ yields

$$
\cos (0)=\cot ^{2}(\tau / 2)(1+\cos (2 \pi / n))-1
$$

and subsequently

$$
2=\cot ^{2}(\tau / 2)(1+\cos (2 \pi / n)) .
$$

Simplifying so that $n$ is on the left-hand side and $\tau$ is on the right gives

$$
\cos (2 \pi / n)=2 \tan ^{2}(\tau / 2)-1,
$$

which indicates the angle measure $\tau$ of a neutral $n$-gon table.
We have essentially proven the following result.

Theorem. Let $P$ be a regular $n$-gon table in the hyperbolic plane with interior angle $\tau$. Then $P$ is

- small when

$$
\cos (2 \pi / n)<2 \tan ^{2}(\tau / 2)-1
$$

- $\quad$ neutral when

$$
\cos (2 \pi / n)=2 \tan ^{2}(\tau / 2)-1, \text { and }
$$

- large when

$$
\cos (2 \pi / n)>2 \tan ^{2}(\tau / 2)-1 \text {. }
$$

We can look for agreement of this theorem with the previously discussed method for classifying triangular tables from [2]. If the table is a triangle with interior angles $\alpha_{1}, \alpha_{2}$, and $\alpha_{3}$, then the table is neutral when

$$
\cos ^{2} \alpha_{1}+\cos ^{2} \alpha_{2}+\cos ^{2} \alpha_{3}+2 \cos \alpha_{1} \cos \alpha_{2} \cos \alpha_{3}=1+\sin \alpha_{1} \sin \alpha_{2} \sin \alpha_{3}
$$

This triangular method overlaps with the theorem above when the table is a regular triangle, that is, when $\tau=\alpha_{1}=\alpha_{2}=\alpha_{3}$. So using the triangular classification method, the table is neutral when

$$
3 \cos ^{2} \tau+2 \cos ^{3} \tau=1+\sin ^{3} \tau
$$

By the theorem, a regular triangular table with interior angle $\tau$ is neutral when 


$$
\cos (2 \pi / 3)=2 \tan ^{2}(\tau / 2)-1
$$

Solving for $\tau$ gives $\tau=2 \arctan (1 / 2)$, which can be substituted into Equation 2, yielding

$$
3 \cos ^{2}(2 \arctan (1 / 2))+2 \cos ^{3}(2 \arctan (1 / 2))=1+\sin ^{3}(2 \arctan (1 / 2)) .
$$

This simplifies to

$$
3\left(\frac{3}{5}\right)^{2}+2\left(\frac{3}{5}\right)^{2}=1+\left(\frac{4}{5}\right)^{3}
$$

and we see that the two classification methods correspond exactly.

As an extra treat, let us apply the theorem to regular 4-gon tables. By solving for $\tau$ we see that the table is small when

$$
\begin{aligned}
& t>2 \arctan (\sqrt{2} / 2), \text { neutral when } \\
& t=2 \arctan (\sqrt{2} / 2), \text { and large when } \\
& t<2 \arctan (\sqrt{2} / 2) .
\end{aligned}
$$

This case is depicted in Figure 7.

\section{CONCLUSION}

In this article we have presented a modest introduction to outer billiards and review of work related to the MoserNeumann problem. We also developed a method for classifying regular polygonal tables in the hyperbolic plane, with large tables having the property that all orbits escape to infinity. Thus the Moser-Neumann problem, which had previously been solved for Penrose kite tables in the Euclidean plane [11] and triangular tables in the hyperbolic plane [2], has been solved for regular polygonal tables in the hyperbolic plane as well. Needless to say, much work remains. For example, is there a way to classify an arbitrary polygonal table in the hyperbolic plane? We hope that interested readers have recognized in outer billiards the beauty that we see and have realized that, although it can get mathematically heavy quite quickly, it is a viable area of research for undergraduates.

\section{ACKNOWLEDGMENTS}

This work was supported in part by a professional development grant from the National Science Foundation Advance Paid grant at Grand Valley State University. We thank the Student Summer Scholars program at Grand Valley State University for its generous support.

\section{REFERENCES}

1. A. Baxter and R. Umble, "Periodic orbits of billiards on an equilateral triangle" Amer. Math. Monthly 115 (2008) 479491.

2. F. Doğru and S. Tabachnikov, "On polygonal dual billiard in the hyperbolic plane" Reg. Chaotic Dynamics 8 (2003) 67-82.

3. E. Gütkin and N. Simanyi, "Dual billiards and necklace dynamics" Comm. Math. Physics. 143 (1991) 431-450.

4. B. Hasselblatt and A. Katok, A First Course in Dynamics, Cambridge Univ. Press, 2003.

5. R. Liboff and M. Porter, "Quantum chaos for the radially vibrating spherical billiard" Chaos 10 (2000) 366-370.

6. A. Lopes and R. Markarian, "Open billiards: Invariant and conditionally invariant probabilities on Cantor sets" SIAM J. App. Math. 56 (1996) 651-680.

7. J. Moser, Stable and Random Motions in Dynamical Systems: With Special Emphasis on Celestial Mechanics, Princeton Univ. Press, 2001.

8. J. Moser, "Is the solar system stable?" Math. Intell. 1 (1978) 65-71.

9. B. Neumann, "Sharing ham and eggs" lota, Manchester University, 1959.

10. S. Otten and F. Doğru, "A lesson learned from outer billiard derivates" Amer. J. Undergraduate Research 9 (2010) 1-7.

11. R. Schwartz, "Unbounded orbits for outer billiards I" J. of Modern Dynamics 1 (2007) 371-424.

12. S. Tabachnikov, Billiards, Soc. Math. de France, 1995.

13. F. Vivaldi and A. Shaidenko, "Global stability of a class of discontinuous dual billiards" Comm. Math. Physics 110 (1987) 625-640.

14. W. Weaver, "The mathematical manuscripts of Lewis Carroll" Proc. Amer. Phil. Soc. 98 (1954) 377-381. 